\title{
WINDOW CONTAMINATION DETECTION METHOD FOR THE ROBOTIC BUILDING MAINTENANCE SYSTEM
}

\author{
Min-sung Kang ${ }^{1}$, Seunghoon Lee², Boill Chun ${ }^{2}$, Kyushik Shin², A. E. Traver ${ }^{3}$, and Chang-soo Han ${ }^{2 *}$ \\ ${ }^{1}$ Department of Mechatronics Engineering, Hanyang University, Korea \\ ${ }^{2}$ Department of Mechanical Engineering, Hanyang University, Korea \\ ${ }^{3}$ Department of Mechanical Engineering, Cockrell School of Engineering, the University of Texas at Austin, USA \\ *Corresponding author (cshan@hanyang.ac.kr)
}

The robotic high-rise building maintenance system is an automated system of performing maintenance works on the outer walls of high-rise buildings, including cleaning, coating, and inspection. This study, a robotic high-rise building maintenance system is proposed that overcomes the conventional limitations and introduces an algorithm for contamination measurement that uses an optical (IR) sensor; quantitative contamination evaluation based on the algorithm; and a strategy for the maintenance of the outer walls of high-rise buildings.

Keywords: Robotic Building Maintenance System, Window Contamination Measuring

\section{INTRODUCTION}

The robotic high-rise building maintenance system is an automated system of performing maintenance works on the outer walls of high-rise buildings, including cleaning, coating, and inspection. The system comprehensively includes a system that minimizes human labor for such labor-intensive works; that reduces accidents at the construction site, including falling and dropping accidents; and that improves the process efficiency and economic feasibility. Existing robotic systems for outer-wall maintenance of high-rise and normal buildings have limitations in that they require significant human labor for supplying resources for the maintenance device, and for installing the device on the outer wall of a building or removing it. As a result, the systems have low efficiency, economic feasibility, and safety [1], [2].

Herein this study, a robotic high-rise building maintenance system is proposed that overcomes the aforementioned limitations. In addition, this study introduces an algorithm for contamination measurement that uses an optical (IR) sensor; quantitative contamination evaluation based on the algorithm; and a strategy for the maintenance of the outer walls of high-rise buildings.

\section{WINDOW CONTAMINATION MEASUREMENT METHODOLOGY FOR BUILDING MAINTENANCE}

A built-in mechanism was used for the robotic building maintenance system to ensure stability. The method of

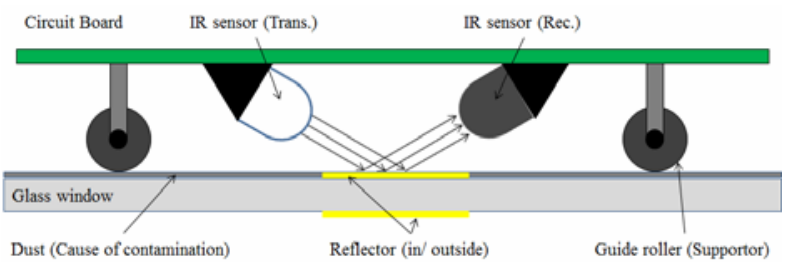

(a) Transient-response area according to the reflector

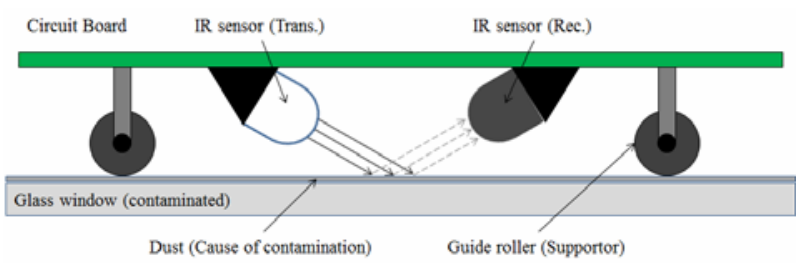

(b) Normal area according to the contamination

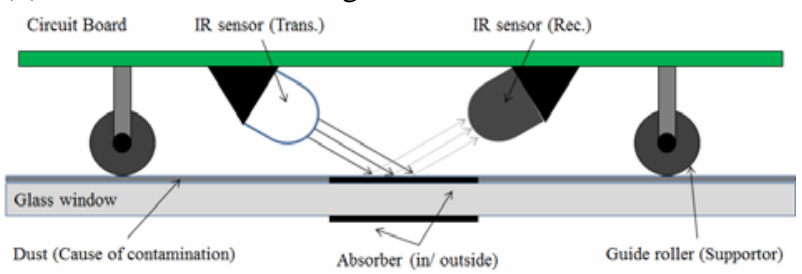

(c) Below-normal area according to the absorber

Fig. 1 Window contamination detecting method 


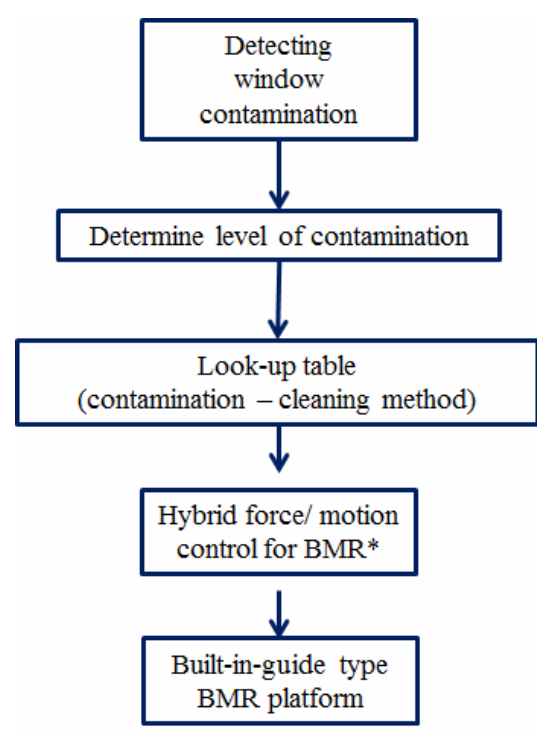

BMR*: Building Maintenance Robot

Fig. 2 Contamination measurement algorithm for outer building wall maintenance

measuring the contamination of outer windows of high-rise buildings for the efficient operation of the system is described as follows (Fig. 1). The contamination measurement device has a sensor module that consists of IR sensors connected in parallel and a clearance roller, which maintains the gap between the window surface and the IR sensor. The device identifies the contamination based on the minimal voltage change according to the window surface contamination (Fig. 2). Among the contaminant areas on the window surface, the transientand below-normal-response areas according to the reflector or absorber were filtered, and the results were used to measure the actual contamination.

\section{EXPERIMENT AND CONCLUSION}

Before measuring the contamination on the window, a
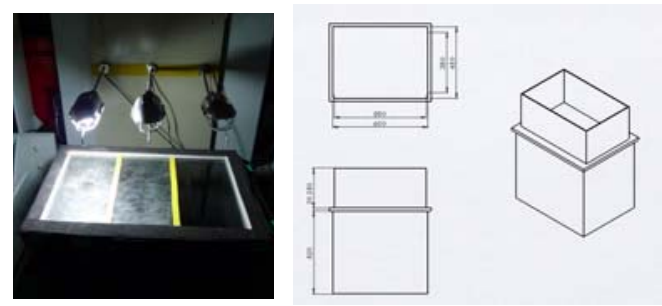

Fig. 3 Experiment configuration for contamination measurement

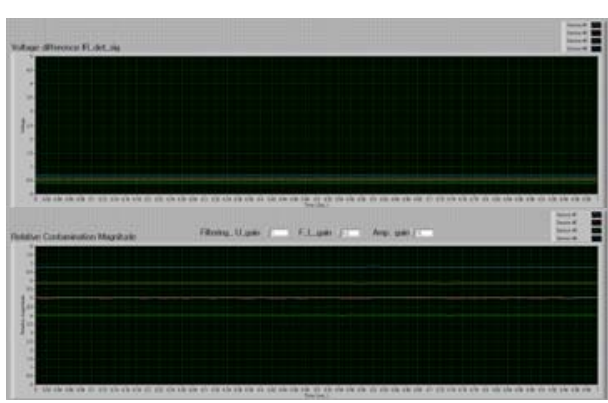

Fig. 4 Relative window contamination measurement results

lighting source was installed on the surface of the outer window to quantitatively evaluate the contamination. Based on the quantity of light that was passing through the window, the contamination was identified.

According to the five-point method, the contamination was evaluated and classified into high/medium/low levels. The illuminance was 35 lux for high-level contamination (dirty window); 27 lux for medium-level contamination; and 19 lux for low-level contamination (clean window). The minimal voltage change appeared according to the contamination level, and the signals were filtered based on the aforementioned algorithm. Weights were given to the relative voltages according to the contamination level, and the results were evaluated as follows (Fig. 4).

\section{ACKNOWLEDGEMENT}

This work was supported by a grant from the Construction Technology Innovation Program (CTIP) funded by the Ministry of Land, Transportation, and Maritime Affairs of Korea (MLTM).

\section{REFERENCES}

[1] A. Albagul, A. Asseni, O. Jomah, M. Omer, B. Farge, "Design and fabrication of an automatic window cleaning robot”, Proceedings of the 9th WSEAS international conference on sigmal processing, roboticds and automation, 2010.

[2] H. Zhang, J. Zhang, R. Liu, W. Wang, G. Zong, “Sky cleaner 3: Pneumatic Climbing robots for glass wall cleaning”, Climbing and walking robots, Vol. 9, pp. 10611069, 2005.Bell, J., “A Mixed-use Renaissance”, Mortgage Banking, Vol. 5(8), pp. 66, 2004. 